\title{
Epidemiology, Incidence, Mortality of Cervical Cancer in Southeast Asia and their Relationship: An Update Report
}

\author{
Nam Hoang Dang Phan, Tung Thanh Nguyen, Nhan Thanh Vo, Trinh Tuyet Thi Le, \\ Tran Bao Hoang Tran, Phuong Kim Truong, Thuy Ai Huyen Le, Thuan Duc Lao*
}

Faculty of Biotechnology, Ho Chi Minh City Open University, Ho Chi Minh City, Vietnam; thuan.ld@ou.edu.vn

\begin{abstract}
Cervical cancer is the leading-caused cancer death in women worldwide, especially occurring in the developing countries. The understanding of the incidence, mortality, and their relationship with the Human Development Index (HDI) and its three dimensions, including gross national income per capita, education index, and life expectancy, is crucial to establish the best way to prevent the increasing of cervical cancer in future. The data of the incidence (-ASR), mortality (-ASR), HDI were extracted from the GLOBOCAN and Human Development Reports database. Person Correlation Coefficient was applied to characterize the relationship among them. The incidence and mortality of Cervical Cancer in Southeast Asia (total new cases: 62,456 cases, counting for 19, 81\%; new death cases: 35,738, counting for $21.22 \%$ ), ranked in the top three of Asian regions. There was the negative correlation between the incidence-ASR, mortality-ASR with HDI, and its three dimensions. A significant correlation between the mortality-ASR rate of cervical cancer and Life expectancy at birth was recorded. The cancer of cervix gravitates to Asian region, including Southeast Asian countries. There was a significant relationship between the mortality-ASR rate of cervical cancer and Life expectancy at birth.
\end{abstract}

Keywords: Cervical Cancer, Incidence, Mortality, Southeast Asia

\section{Introduction}

Cervical cancer is the most cancer leading-death for women worldwide, especially occurring in the developing countries. It remains the serious public health problem threatening to women health ${ }^{1,2}$. According to the data of the International Agency for Research on Cancer, in 2012, total of 14.1 million new cancer cases, and 8.2 million cancer deaths were detected in round of world. In which, 528,000 new cervical cancer cases and 266,000 cervical cancers caused death was identified. Moreover, the highest ASIR of cervical cancer was gravitated to the Southeast Asia countries, including Myanmar, Singapore, Indonesia, Thailand, Philippines, and Brunei. According to the cervical cancer etiology, certain types of Human
Papilloma-Virus (HPV) are the major cause of invasive cervical cancer and cervical gland inflammation ${ }^{3,4}$. Besides that many other etiological factors, including the genetic modification, epigenetic alterations, lifestyles, etc. have been reported to play key roles in cervical tumorigenesis $\mathrm{s}^{3,4}$.

Human Development Index (HDI) is an index that measures the three key dimensions of human development: gross national income per capita, education index and life expectancy index ${ }^{5,6}$. HDI is classified into four levels, including low $(\mathrm{HDI}<0.535)$, medium $(0.710>\mathrm{HDI}>0.535)$, high $(0.80>\mathrm{HDI}>0.710)$ and very high (HDI $>0.80$ ) level ${ }^{5-8}$. Up to date, the classification of HDI has been used to perform the comparison of the incidence, mortality of much human disease, including

${ }^{*}$ Author for correspondence 
cervical cancer. The understanding of association among these criteria could lead to the accurate understanding of the distribution of human cancers. Notably, the incidence and mortality of cervical cancer are annually increasing in the Southeast Asian countries, thus, the reported information of the incidence and mortality of cervical cancer seem to be very important to open up the range of cancer prevention. Hence, in current study, we reported the incidence, mortality of cervical cancer, and its association with the HDI, its components in Southeast Asia countries in the data updated to the year of 2018.

\section{Methods}

\subsection{Study Design}

The current ecological study is done to present and evaluate the correlation between HDI index, its components, including gross national income per capita, education index, and life expectancy, with the incidence and mortality rate of the cervical cancer in Southeast Asian countries 5 . Data of age-specific incidence and mortality of cervical cancer in 2018 was obtained from the global cancer observatory for each countries in Southeast Asia, available from http://gco.iarc.fr/today/ home9. Data of HDI and its components were collected from Human Development Reports database, United Nations Development Program, available from http://hdr. undp.org/en/data ${ }^{10}$.

\subsection{Statistical Analysis}

In current study, the Pearson Correlation Coefficient method was performed to assess the correlation between ASR with HDI and its components. Statistical significance was assumed if the $\mathrm{p}$-value $<0.05$.

\section{Results}

\subsection{Finding Cervical Cancer Case, HDI and its Components}

In 2018, total of 8,622,539 new cases cancer and 4,169,387 new cancer caused death were recorded in female worldwide. Of which, 569,847 (counting for 6.61\%) new cancer of cervix cases and 311,365 (counting for 7.47\%) cervical cancer caused death were recorded. Concerning to the cervical cancer, which has remarkably pronounced differences in distribution according to geography and ancestry, gravitating toward Asian countries (Total new cases: 315,346 cases (counting for $55.34 \%$ ), new death cases: 168,411 cases (counting for $54.09 \%$ )). In this study, the authors represented the epidemiology, incidence and mortality of Cervical Cancer in Southeast Asia (total new cases: 62,456 cases, counting for $19.81 \%$; new death cases: 35,738 , counting for $21.22 \%$ ), ranked in the top three of Asian regions. In Southeast Asian countries, the top five countries, which have the highest incidence, are Indonesia (32,469 new cases, counting for 51.99\%), Thailand (8,622 new cases, counting for $13.80 \%$ ), Philippines (7,190 new cases, counting for $11.51 \%)$, Myanmar (6,472 new cases, counting for $10.36 \%)$ and Viet Nam (4,177 new cases, counting for $6.69 \%)$. According to the mortality of cervical cancer, the top five countries are (1) Indonesia $(18,279$ new cases, counting for 51.15\%), (2) Thailand (5,015 cases, counting for $14.03 \%)$, (3) Philippines (4,088 cases, counting for $11.44 \%)$, (4) Myanmar (3,856 cases, counting for $10.79 \%)$ and (5) Viet Nam (2,420 cases, counting for 6.77\%) (Table 1). Therefore, based on these data, the top five Southeast Asian countries with the highest incidence and mortality of cervical cancer are Indonesia, Thailand, Philippines, Myanmar and Viet Nam.

The Age-world-standardized incidence rate (incidenceASR), crude incidence, the Age-world-standardized mortality rate (mortality-ASR), and crude mortality of cervical cancer in Southeast Asian countries were shown in (Table 2\&3). The top five countries, which have the highest incidence-ASR, are Indonesia: Indonesia 23.4 per 100,000 people, Myanmar: 21.5 per 100,000 people, Brunei: 20.6 per 100,000 people, Thailand: 16.2 per 100,000 people, and Philippines: 14.9 per 100,000 people. The five countries with the highest mortality-ASR are: Indonesia: 13.9 per 100,000 people; Myanmar: 13.1 per 100,000 people; Cambodia: 10.1 per 100,000 people; Thailand: 9.0 per 100,000 and Philippines: 8.8 per 100,000 .

The values of HDI and its components of Southeast Asian countries were shown in (table 4). Of which, Singapore is the country with the highest HDI of 0.932 . According to HDI, Southeast Asian countries were classified in three categories of human develop level as follow: three countries in very high human develop category, includes Singapore, Brunei, Malaysia; one country in high category, includes Thailand; seven countries in medium category, includes Philippines, Indonesia, Viet Nam, Timor-Leste, Lao People's Democratic Republic, Cambodia and Myanmar. 
Table 1. The incidence and mortality of cervical cancer of the Southeast Asian countries in 2018 (sorted by the incidence rate and mortality rate from the highest to lowest value)

\begin{tabular}{|c|c|c|c|c|c|c|c|c|}
\hline \multirow{2}{*}{ Countries } & \multicolumn{4}{|c|}{ Incidence } & \multicolumn{4}{c|}{ Mortality } \\
\cline { 2 - 10 } & Cases & Rank & \% & Cum.risk & Cases & Rank & \% & Cum.risk \\
\hline Indonesia & 32,469 & 1 & 51.99 & 14.3 & 18,279 & 1 & 51.15 & 1.6 \\
\hline Thailand & 8,622 & 2 & 13.80 & 16.3 & 5,015 & 2 & 14.03 & 1.0 \\
\hline Philippines & 7,190 & 3 & 11.51 & 16.5 & 4,088 & 3 & 11.44 & 1.0 \\
\hline Myanmar & 6,472 & 4 & 10.36 & 13.9 & 3,856 & 4 & 10.79 & 1.5 \\
\hline Viet Nam & 4,177 & 5 & 6.69 & 15.6 & 2,420 & 5 & 6.77 & 0.47 \\
\hline Malaysia & 1,682 & 6 & 2.69 & 14.5 & 944 & 6 & 2.64 & 0.72 \\
\hline Cambodia & 993 & 7 & 1.59 & 13.1 & 708 & 7 & 1.98 & 1.2 \\
\hline Singapore & 429 & 8 & 0.69 & 23.3 & 208 & 8 & 0.58 & 0.40 \\
\hline Lao People's Democratic Republic & 320 & 9 & 0.51 & 16.2 & 182 & 9 & 0.51 & 0.82 \\
\hline Brunei & 52 & 10 & 0.08 & 21.2 & 14 & 11 & 0.04 & 0.77 \\
\hline Timor-Leste & 50 & 11 & 0.08 & 9.2 & 24 & 10 & 0.07 & 0.73 \\
\hline Total & 62,456 & - & 100.00 & & 35,738 & - & 100.00 & \\
\hline
\end{tabular}

Table 2. The Age-world-standardized incidence rate (incidence-ASR), crude incidence of cervical cancer in Asian Countries

\begin{tabular}{|c|c|c|c|}
\hline Countries & Incidence-ASR & Rank & Crude rate \\
\hline Indonesia & 23.4 & 1 & 24.5 \\
\hline Myanmar & 21.5 & 2 & 23.5 \\
\hline Brunei & 20.6 & 3 & 24.7 \\
\hline Thailand & 16.2 & 4 & 24.3 \\
\hline Philippines & 14.9 & 5 & 13.6 \\
\hline Cambodia & 13.5 & 6 & 11.9 \\
\hline Timor-Leste & 12.5 & 7 & 7.7 \\
\hline $\begin{array}{c}\text { Lao People's } \\
\text { Democratic } \\
\text { Republic }\end{array}$ & 11.4 & 8 & 9.2 \\
\hline Malaysia & 10.5 & 9 & 10.8 \\
\hline Singapore & 7.7 & 10 & 14.6 \\
\hline Viet Nam & 7.1 & 11 & 8.6 \\
\hline
\end{tabular}

\subsection{Evaluating the Association between Incidence-ASR, Mortality-ASR and HDI}

The analysis results indicated the weak negative correlation between the incidence-ASR and HDI ( $\mathrm{R}$ $=-0.19$ ) (Figure 1), the moderate negative correlation between the mortality-ASR and HDI $(\mathrm{R}=-0.53)$ (Figure 2 ). However, these relationships were no statistically significant $(\mathrm{p}>0.05)$. There was also a negative correlation between the three dimensions of HDI with morbidity and mortality of cervix cancer (Table 4). Only the correlation
Table 3. The Age-world-standardized morality rate (morality-ASR), crude morality of cervical cancer in Asian Countries

\begin{tabular}{|c|c|c|c|}
\hline Countries & Mortality-ASR & Rank & Crude rate \\
\hline Indonesia & 13.9 & 1 & 13.8 \\
\hline Myanmar & 13.1 & 2 & 14.0 \\
\hline Cambodia & 10.1 & 3 & 8.5 \\
\hline Thailand & 9.0 & 4 & 14.1 \\
\hline Philippines & 8.8 & 5 & 7.7 \\
\hline $\begin{array}{c}\text { Lao People's } \\
\text { Democratic } \\
\text { Republic }\end{array}$ & 7.0 & 6 & 5.2 \\
\hline Timor-Leste & 6.2 & 7 & 3.7 \\
\hline Brunei & 6.1 & 8 & 6.6 \\
\hline Malaysia & 6.0 & 9 & 6.1 \\
\hline Viet Nam & 4.0 & 10 & 5.0 \\
\hline Singapore & 3.8 & 11 & 7.1 \\
\hline
\end{tabular}

between mortality-ARS and Life expectancy at birth index was statistically significant $(\mathrm{p}=0.02)$ (Table 4$)$.

\section{Discussion}

According to the data of GLOBOCAN, cervical cancer is the fourth most common cause of cancer incidence and mortality in women ${ }^{9,11}$. Southeast Asia contributes is one of top three regions that have the highest cervical cancer incidence (contributed to 55.34\%), and mortality 


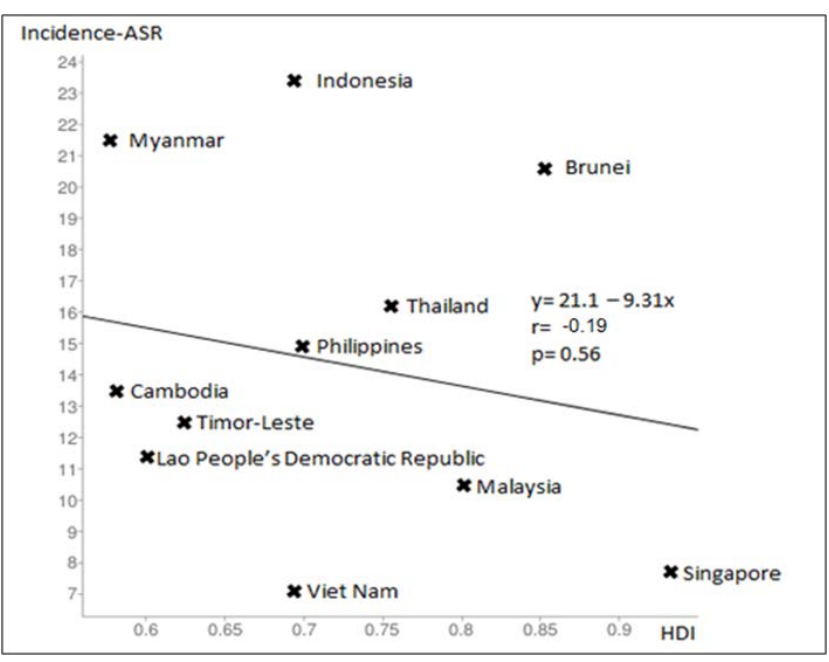

Figure 1. Correlation between HDI and incidence-ASR of cervical cancer in Southeast Asian countries in the year of 2018.

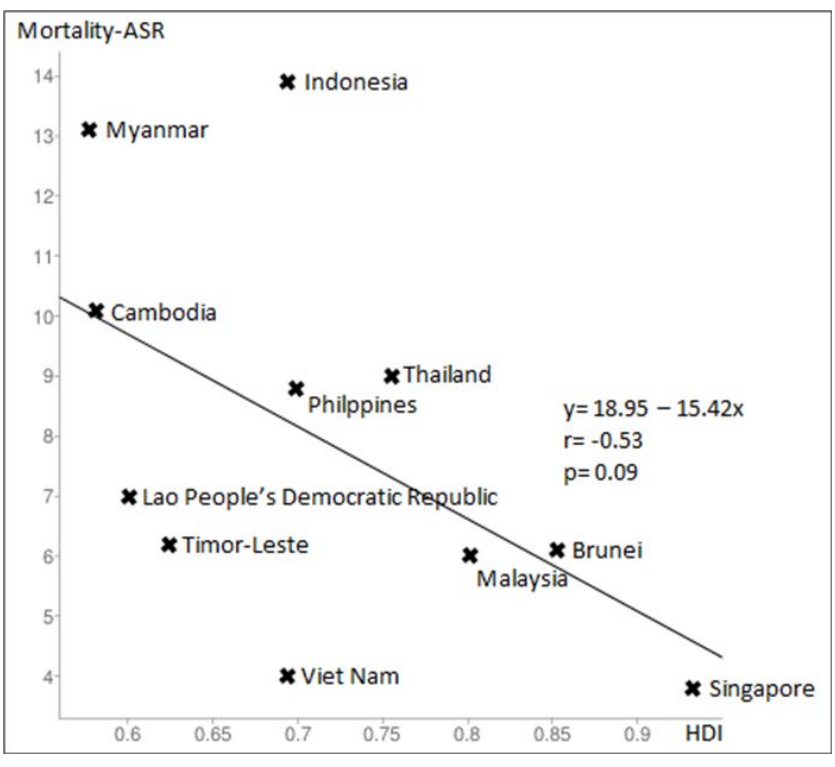

Figure 2. Correlation between HDI and mortality-ASR of cervical cancer in Southeast Asian countries in the year of 2018 . (contributed to $54.09 \%)^{9}$. The number of cases of cervix uteri cancer in Southeast Asian countries given in GLOBOCAN increased over time (In year of 2012: incidence: 175,229 new cases, mortality: 94,294 cases; in the year of 2018: incidence: 315,346 new cases, mortality: 168,411 cases). The top three regions with highest incidence and mortality of cervical cancer are Eastern Asia, South-central Asia, and Southeast Asia). Thus, the cervical cancer incidence and mortality rate vary considerably between different regions in the world, and has typically the highest rate among the countries of Asian. The differences in the incidence and mortality of cervical cancer among different regions can be attributed to the following factors: the existence, duration, and quality of screening programs ${ }^{12}$.

In this article, the authors found out that there was a negative correlation between incidence-ASR, mortalityASR and HDI, its dimensions, including gross national income per capita, education index, and life expectancy. The linear regression model showed that with increasing HDI, and its three dimensions, including gross national income per capita, education index, and life expectancy, the incidence-ASR and mortality-ASR rate decreased. The top three countries with the highest incidenceASR and mortality-ASR are Indonesia, Myanmar and Cambodia, which are ranked as the medium human development countries. In contrast, the two highest HDI countries: Singapore, Malaysia was the countries within the low incidence and mortality of cervical cancer. In the high-income countries, taking an advantage of good budget allocations for health care and public health sectors, services, it is distributed to the well diagnosis and treatment to reduce the mortality of cervical cancer that result from the treatment of cervical cancers. The linear regression model showed that with increasing education index, mortality and incidence of cervical cancer rates decreased. It can be explained that with increasing

Table 4. HDI and its components in South-east Asian countries in 2018

\begin{tabular}{|c|c|c|c|c|c|}
\hline \multicolumn{2}{|c|}{ Variable } & HDI & Life expectancy at birth & Mean year of schooling & GNI/ capita \\
\hline \multirow{2}{*}{ Incidence-ASR } & $\mathrm{r}$ & $-1,96$ & $-0,42$ & $-0,23$ & $-0,07$ \\
\cline { 2 - 6 } & $\mathrm{p}$ & 0,56 & 0,19 & 0,49 & 0,84 \\
\hline \multirow{2}{*}{ Mortality-ASR } & $\mathrm{r}$ & $-0,53$ & $-0,67$ & $-0,43$ & $-0,48$ \\
\cline { 2 - 6 } & $\mathrm{p}$ & 0,09 & 0,02 & 0,19 & 0,13 \\
\hline
\end{tabular}


knowledge, education, women are more likely to carry health care, and supporting with the development of cancer treatment resulting in mortality decreased. Overall, it could be stated that following criteria, including unhealthy lifestyles, such as cigarettes, culture, sexual factors; environmental influences, such as chemicals, pollution; Inflammation, obesity and HPV are the main causes of cervical cancer. In developed countries, due to a lack of medical and diagnostic services, education, the incidence-ASR and mortality-ASR of cervical cancer is high.

\section{Conclusion}

The high incidence and mortality rate of cervical cancer were recorded in Southeast Asian countries (incidence: 315,346 new cases, mortality: 168,411 cases). The cervical cancer incidence and mortality of cervical cancer are increasing over time, compared to the year of 2012. The negative linear aggression indicated that increasing the value of $\mathrm{HDI}$, and its three dimensions, including gross national income per capita, education index, and life expectancy, the incidence-ASR and mortality-ASR rate decreased. Notably, there was a significant relationship between the mortality-ASR rate of cervical cancer and Life expectancy at birth.

\section{Conflict of Interest Statement}

The authors declare that there is no conflict of interest regarding the publication of this paper.

\section{References}

1. Sherris J, Herdman C, Elias C. Cervical cancer in the developing world. West J. Med. 2001; 175(4):231-33. https://doi.org/10.1136/ewjm.175.4.231. PMid: 11577044, PMCid: PMC1071564.

2. Wong LP. Knowledge and attitudes about HPV infection, HPV vaccination, and cervical cancer among rural Southeast Asian Women. Int. J. Behav. Med. 2011; 18(2):105-11. https://doi.org/10.1007/s12529-010-9104-y. PMid: 20524163.
3. Mohanty G, Ghosh SN. Risk factors for cancer of cervix, status of screening and methods for its detection. Arch. Gynecol. Obstet. 2015; 291(2):247-49. https://doi. org/10.1007/s00404-014-3492-1. PMid:25273981.

4. Muñoz N, Bosch FX, De Sanjosé S, et al. Epidemiologic classification of human Papillomavirus types associated with cervical cancer. N. Engl. J. Med. 2003; 348(6):518-27. https://doi.org/10.1056/NEJMoa021641. PMid: 12571259.

5. Lao D, Le H. Epidemiology, incidence and mortality of Nasopharynx Cancer in Southeast Asia: An update report. Adv. Life Sci. 2020; 7(2):86-90.

6. Bray F, Jemal A, Grey N, Ferlay J., Forman, D. Global cancer transitions according to the Human Development Index (2008-2030): A population-based study. Lancet Oncol. 2012; 13(8):790-01. https://doi.org/10.1016/S14702045(12)70211-5.

7. Soheylizad M, Khazaei S, Jenabi E, Delpisheh A, Veisani Y. The relationship between human development index and its components with thyroid cancer incidence and mortality: Using the decomposition approach. Int. J. Endocrinol. Metab. 2018; 16(4):e65078. https://doi.org/10.5812/ ijem.65078. PMid: 30464773, PMCid: PMC6218660.

8. Mahdavifar N, Towhidi F, Makhsosi BR, et al. Incidence and mortality of Nasopharynx cancer and its relationship with human development index in the world in 2012. World J. Oncol. 2016; 7(5-6):109-18. https://doi.org/10.14740/ wjon980w. PMid:28983375 PMCid:PMC5624652.

9. Cancer Today. Cancer Fact sheet. Accessed 12th December, 2019. Available from https:/gco.iarc.fr/today/fact-sheetscancers.

10. United Nations Development Programme. Human Development Data (1990-2018). Accessed 12th December 2019. Available from http://hdr.undp.org/en/data.

11. Arbyn M, Weiderpass E, Bruni L, et al. Estimates of incidence and mortality of cervical cancer in 2018: A worldwide analysis. Lancet Glob Heal, 2020; 8(2):e191-03. https://doi.org/10.1016/S2214-109X(19)30482-6.

12. Momenimovahed $Z$, Salehiniya $H$. Incidence, mortality and risk factors of cervical cancer in the world. Biomed. Res. Ther. 2017; 4(12):1795-811. https://doi.org/10.15419/ bmrat.v4i12.386. 